\title{
24-hour Holter findings in infants with in utero exposure to Zika virus: a cross-sectional study.
}

Dulce Helena Gonçalves Orofino ( $\nabla$ dulceorofino@yahoo.com.br)

Instituto Fernandes Figueira https://orcid.org/0000-0002-0953-1831

Sonia Regina Lambert Passos

Fundacao Oswaldo Cruz

Sheila Moura Pone

Instituto Fernandes Figueira

Marcos Silva Pone

Instituto Fernandes Figueira

Elisa Barroso de Aguiar

Instituto Fernandes Figueira

Igo Oliveira de Araújo

Instituto Fernandes Figueira

Thiago Moreira Ramos

Universidade Estacio de Sa - Campus Reboucas-Rio Comprido

Leticia Machado Lima e Silva

Instituto Fernandes Figueira

Bruna Menezes

Universidade Estacio de Sa Faculdade de Medicina

Luan Noé da Silva

Fundacao Oswaldo Cruz

Rafaela Valentim Goldenzon

Fundacao Tecnico-Educacional Souza Marques Escola de Medicina Souza Marques

Raffaela Andrade de Oliva

Universidade Estacio de Sa Faculdade de Medicina

Maria Elizabeth Moreira

Instituto Fernandes Figueira

Raquel de Vasconcellos Carvalhaes de Oliveira

Fundacao Oswaldo Cruz

Research article

Keywords: Zika virus, Holter, arrhythmias, heart rate variability 
Posted Date: September 24th, 2019

DOl: https://doi.org/10.21203/rs.2.14894/v1

License: (c) (i) This work is licensed under a Creative Commons Attribution 4.0 International License. Read Full License 


\section{Abstract}

Background : Antenatal exposure to the Zika virus (ZIKV) is related to severe neurological, ophthalmological and orthopedic manifestations called congenital Zika syndrome (CZS), but no changes in the electrical activity of the heart have been found. A higher prevalence of structural cardiac changes has been described in CZS patients when compared to the general population of live births. To describe 24-hour Holter findings in infants with confirmed antenatal exposure to ZIKV.

Methods : Cross-sectional study of 24-hour Holter findings in infants with antenatal exposure to ZIKV confirmed by positive polymerase chain reaction(PCR). Patients of the cohort of newborns and infants exposed intrautero to the ZIKV which began in November 2015 and continues to date and were hospitalized for clinical or surgical reasons were included. The 24-hour Holter monitoring was performed the day before discharge. Data analysis was descriptive and included subgroups of patients according to the presence of CZS and severe microcephaly. Heart rate, ST segment, QT interval and arrhythmias were evaluated. To evaluate heart rate variability (HRV) we used time domain analysis. The Mann-Whitney test was performed to verify significant differences between the subgroups of patients.

Results : The sample consisted of 15 infants with average age of 16 months and 12 were diagnosed with CZS (8 had severe microcephaly). PCR at birth was positive in 8 newborn and 7 had positive maternal PCR during pregnancy. No arrhythmias or QT interval changes were observed. The Mann-Whitney test showed a significant difference between patients with and without CZS and with and without severe microcephaly, with HRV being lower in the groups with severe microcephaly and CZS.

Conclusions : The study suggests that the HRV evaluation can be used as a sentinel event to assess the possibility of progressive neurological impairment in newborns with antenatal exposure to ZIKV, allowing early initiation of stimulation therapies in addition to indicating that cardiovascular complications may arise in the medium and long term.

\section{Background}

The Zika virus (ZIKV) epidemic that occurred from the beginning of 2015 was accompanied by severe damage to the central nervous system (CNS) and other organs of some fetuses exposed to ZIKV in the intrauterine period. Fetal ultrasound findings such as microcephaly was reported in $82.3 \%$ of cases, and newborn skull tomography findings such as ventriculomegaly (93.8\%), cerebral calcifications $(98.8 \%)$, and poorly developed cerebellum or cerebellar vermis (51.8\%) were reported[1]. Congenital Zika syndrome (CZS) is defined by the combination of microcephaly, specific changes in CNS imaging (computed tomography), ophthalmic alterations, and arthrogryposis[2]. Despite not composing CZS, recent studies have evaluated findings from Doppler two-dimensional echocardiograms of newborns and infants with confirmed antenatal exposure to ZIKV and showed a 10-fold higher prevalence of structural alterations than in the general population of live births. The most common heart diseases were pulmonary hyperflow 
(interatrial and interventricular communication and patent ductus arteriosus). No serious cardiopathy was detected with indications for drug or surgical therapy in the first months of life[3,4].

The autonomic nervous system (ANS) and the balance of its sympathetic and parasympathetic arms regulate cardiovascular function during intrauterine and postnatal life[5]. Studies relate the imbalance between the sympathetic and parasympathetic ANS with the development of systemic hypertension, cardiovascular diseases, diabetes mellitus, and asthma as well as cognitive and behavioral changes[6].

Thorough knowledge of the anatomy and development of the ANS has allowed a better understanding of its involvement in cardiac arrhythmias. The literature suggests that ANS dysregulation caused by gene polymorphisms that modulate ANS development may be implicated in sudden infant death syndrome (SIDS). Furthermore, an imbalance between the sympathetic and parasympathetic ANS can lead to cardiac arrhythmias, and the predominance of one over the other may vary depending on the type of arrhythmia. For example, in Brugada syndrome, arrhythmic events have been associated with periods of higher parasympathetic tone and in catecholaminergic polymorphic ventricular tachycardia, ventricular arrhythmia events are associated with the predominance of the sympathetic ANS[7].

The gold standard for studying the electrical activity of the heart is 24-hour Holter monitoring, which records the electrical activity of the patient in usual routine for 24 hours[8]. Fluctuations in heart rate (HR) are related to neural activity, and HR variability (HRV) that is obtained through 24-hour Holter monitoring is the end result of the integrated and rhythmic activity of ANS neurons generated by organized cardiorespiratory reflexes being recognized as a sign of heart health[9,10]. HRV assessment is performed using time domain measures such as mean R-R values (measured between 2 consecutive $R$ waves of the trace), SDNN (standard deviation of R-R intervals throughout the Holter "clean trace" time), pNN50 (the mean number of times per hour when the R-R intervals exceed 50 milliseconds) and rMMSD (the mean square root of successive differences between normal heart beats) and by frequency domain measurements (low, high and very high) after thesoftware analyzing the exam has deleted the trace periods with interference. The heart rate increases rapidly in the first week of life and then decreases, and HRV decreases in this early neonatal period (following the highest HR) and then increases, indicating a predominance of the parasympathetic ANS over the sympathetic ANS after 6 months of age[9,11]. Studies conducted since the 1980s have suggested that HRV assessment may be a useful tool to provide information about neurodevelopment in children exposed to risks of perinatal brain damage because HRV would be related not only to the functional integrity of the ANS but to the entire central nervous system (CNS) $[12,13]$. Lower HRV has also been reported in patients with treatment-refractory epilepsy, suggesting that this may explain the higher occurrence of sudden death in these patients[14].

To date there are no data related to changes in the electrical activity of the heart of the infants with antenatal exposure to ZIKV and CZS. In view of these findings and the importance of HRV in assessing the integrity of the nervous system, 24-hour Holter monitoring is justified in patients with confirmed antenatal exposure to ZIKV due to potential CNS impairment in these patients. 


\section{Methods}

Fifteen patients of the cohort of newborns and infants with antenatal exposure to the ZIKV which began in November 2015 and continues to date, at the Instituto Fernandes Figueira (IFF), Rio de Janeiro, Brazil and were hospitalized for clinical or surgical reasons were included. The 24-hour Holter monitoring was performed the day before discharge. Antenatal exposure was confirmed by positive polymerase chain reaction (PCR) of ZIKV from the mothers' blood samples during pregnancy and/or in the newborn urine or cerebrospinal fluid right after birth[15]. The sample was divided into subgroups, according to the following characteristics: sex (female and male); positive or negative PCR for ZIKV in the newborn; with or without a history of fetal distress (Apgar score less than 6 at 5 minutes of life)[16]; preterm (PT) (gestational age less than 36 weeks and 6 days) or not PT[17]; small for gestational age (SGA) or not SGA (according to the definition established in the literature)[16]; microcephaly (head perimeter (HP) less than two standard deviations (SD) for age, sex and gestational age), severe microcephaly (HP less than three SD for age, sex and gestational age) or no microcephaly[18]; and with or without CZS[2]. The parents were instructed to fill out an activity record during the 24 hours, but because most of the patients had CNS involvement, there was no report of physical activity (walking, running) in 14 of the 15 patients in the sample (only 1 patient had no impairment of motor functions); therefore, 14 patients remained in their beds throughout the examination period.

Using a DMS 300 device, Satellite Resting v.78 model, and analysis of 3 leads, continuous monitoring was performed for 24 hours with the patient in his or her usual routine. According to the guidelines of the Task Force of the European Society of Cardiology and the North American Society of Pacing and Electrophysiology[19], only tests with more than 18 hours of "clean trace" were analyzed. To determine HRV parameters, Holter software was used, and all traces were subsequently analyzed individually by two cardiologists who specialize in Holter analysis. The complexes classified as "noise" or with artifacts were manually excluded. Maximum, minimum and mean HR, pauses, presence of supraventricular and ventricular extrasystoles, bigeminal or trigeminal events, ST segment and QT changes were evaluated. The HRV assessment was performed through 24 hour R-R and SDNN, pNN50 and rMMSD. A nonparametric Mann-Whitney test was used to evaluate differences in median values among the groups of patients, using p-values $<0.05$ as suggestive of a significant difference. Additionally, we added measures of uncertainty (such as, minimum, maximum and range) due to sample size and the critical to exclusive use of $\mathrm{p}$-values[20].

Patients who presented with some alteration in the 24-hour Holter assessment were included in the IFF Cardiopediatrics outpatient clinic.

\section{Results}

The sample consisted of 15 patients (9 males) with a mean age of 16 months. Of the 15 patients in the sample, 4 were hospitalized only for Holter and 11 patients were hospitalized for one or more clinical and / or surgical reasons (three due to difficult to control seizures, 5 due to swallowing disorder, insufficient 
weight gain and performance, 9 with high or low respiratory conditions, two due to urinary tract infection, one due to ventriculoperitoneal shunt, and one due to gastrostomy ostium infection). All patients were using medications during the exam: 10 patients on anticonvulsivants use-valproic acid, clobazan, topiramate, phenytoin, vigabatrin; 8 patients on oxybutynin use; two patients using clavulanate amoxacillin; 5 on ranitidine use; 4 on betasimpathomimetics and one on oral corticosteroids. The 4 patients who were hospitalized for Holter only used multivitamins. The average sleep time during the 24 hours was 11 hours and 40 minutes. No patient was excluded from the study because had less than 18 hours of Holter clean trace.

There was no record of pauses, junctional rhythm, supraventricular or ventricular extrasystoles, blockages or QT interval alterations. The mean HR was higher among infants with severe microcephaly and CZS, and HRV measurements had lower median values in the groups with CZS and severe microcephaly (Table 1). No difference was detected between the groups with and without positive newborn's PCR and with and without fetal distress and among the groups of born SGA, PT or not. The data show that in the CZS and severe microcephaly groups, there was a significant difference in SDNN values $(p<0.05$ in both groups) and $R-R$ values ( $<<0.05$ in the group with CZS) (Table 1$)$.

Table 1: Median parameters of heart rate variability in 15 infants with antenatal exposure to ZIKV according to subgroup with 95\% confidence interval 


\begin{tabular}{|c|c|c|c|c|c|c|}
\hline \multirow{2}{*}{$\begin{array}{l}\text { Median (Min - } \\
\text { Máx) }\end{array}$} & \multicolumn{3}{|c|}{ Severe microcephaly } & \multicolumn{3}{|c|}{ CZS } \\
\hline & No & Yes & p-value & No & Yes & p-value \\
\hline *HRm & $\begin{array}{c}115 \\
(102-141)\end{array}$ & $\begin{array}{c}137 \\
(109-174)\end{array}$ & 0,0558 & $\begin{array}{c}114 \\
(103-115)\end{array}$ & $\begin{array}{c}139,5 \\
(102-174)\end{array}$ & 0,0601 \\
\hline **pNN50 & $\begin{array}{c}19 \\
(0-25)\end{array}$ & $\begin{array}{c}3,5 \\
(2-18)\end{array}$ & 0,0716 & $\begin{array}{c}19 \\
(10-25)\end{array}$ & $\begin{array}{c}4,5 \\
(0-23)\end{array}$ & 0,0819 \\
\hline ***rMSSD & $\begin{array}{c}43 \\
(9-49)\end{array}$ & $\begin{array}{c}22 \\
(17-39)\end{array}$ & 0,0818 & $\begin{array}{c}44 \\
(31-49)\end{array}$ & $\begin{array}{c}24 \\
(9-47)\end{array}$ & 0,0507 \\
\hline †RR & $\begin{array}{c}515,65 \\
(436,24- \\
604,45)\end{array}$ & $\begin{array}{c}446,715 \\
(357,13- \\
560,09)\end{array}$ & 0,0541 & $\begin{array}{c}521,2 \\
(515,65- \\
604,45)\end{array}$ & $\begin{array}{c}459,91 \\
(357,13- \\
593,340)\end{array}$ & $0,0484 *$ \\
\hline$t+S D N N$ & $\begin{array}{c}78 \\
(46-94)\end{array}$ & $\begin{array}{c}59,5 \\
(32-79)\end{array}$ & $0,0205 *$ & $\begin{array}{c}83 \\
(78-86)\end{array}$ & $\begin{array}{c}62,5 \\
(32-94)\end{array}$ & $0,0484 *$ \\
\hline
\end{tabular}

*HRm: mean heart rate in beats per minute

**pNN50:the average number of times per hour where R-R intervals exceed 50 milliseconds

***rMMSD: mean square root of successive differences between normal heart beats

†R-R: interval in milliseconds between 2 consecutive $\mathrm{R}$ waves

†† SDNN: standard deviation of R-R intervals during the entire Holter "clean trace" time

\section{Discussion}


The evaluation of HRV as an instrument to obtain information about neurodevelopment in children with some risk of cerebral damage in the perinatal period was proposed by Bjelakovic et al., who studied 35 infants aged between 2 and 6 months and diagnosed with mild to severe central coordination disorder and 37 healthy controls. Patients in the group with coordination disorders were followed up for 18 months and reevaluated regarding neurological changes (late diagnosis of cerebral palsy). The study suggested that SDNN values $<48$ milliseconds (ms) were predictive of worse neurological outcome and diagnosis of cerebral palsy at 18 months[21]. In our sample, 4 patients with CZS presented SDNN less than $48 \mathrm{~ms}$, and there was a significant difference between the SDNN values for the groups with and without CZS; the values for the group with CZS were lower than those for the group without CZS. This finding may suggest that the evaluation of HRV in young infants with vertical exposure to ZIKV and born without CZS may indicate which of these patients will develop worsening symptoms of delayed neuropsychomotor development during the first year of life. In addition, Galland et al. studied the HRV of 23 newborn SGA patients with idiopathic delayed intrauterine growth at 1 and 3 months, suggesting that the predominance of the sympathetic ANS observed by lower HRV in SGA newborns may be related to an increased risk of hypertension, diabetes mellitus, and other cardiovascular diseases in adulthood[5]. In our sample, only two patients were born SGA, which makes it difficult to analyze the p-value for significant differences between the SGA and non-SGA groups. The finding of lower SDNN in infants with CZS who participated in our study draws attention to other comorbidities that may develop during the life of these patients and to the need for follow-up and measures to prevent cardiovascular diseases.

The study of HRV has also been used to assess the likelihood of sudden infant death syndrome (SIDS), and it has been suggested that lower HRV and higher HR are associated with a higher probability of this outcome[22]. The follow-up of a cohort in the 1990s, composed of 6914 newborns and infants, among whom 16 died from SIDS and who performed 24-hour Holter monitoring in the first months of life, indicated an association of a higher HR and lower HRV with a higher probability of death by SIDS[22]. The evaluation of HRV in this study was performed through the analysis of the mean and standard deviation of the R-R. The study suggested that cardiac instability may cause potentially lethal arrhythmias, and during the period of increased ANS development, which coincides with the period of increased risk of SIDS, some children develop accelerated or asymmetrical development of the sympathetic arm of the ANS, placing them at a higher risk of SIDS. Our study showed a statistically significant difference between R-R values in patients with and without CZS (the CZS group showed a lower R-R value), but our sample was older than that studied in the aforementioned cohort, making it difficult to compare the absolute values.

During monitoring, all patients, except for the 4 patients hospitalized for Holter monitoring only, were taking medications (anticonvulsants, anticholinergics, histamine receptor antagonists, corticosteroids, beta-sympathomimetics and antibiotics). A systematic review and meta-analysis of HRV, epilepsy and antiepileptic drugs showed that patients with difficult-to-control epilepsy who were using more than one anticonvulsant had lower HRV than patients with less than one seizure per month who were on monotherapy. The study draws attention to the increased risk of sudden death in patients with epilepsy and suggests that the use of more than one medication to control seizures may be an independent risk 
factor for the occurrence of sudden death due to autonomic imbalance[23]. We found no study on HRV and the other medications used by our patients. The patients in our sample who were not using anticonvulsants comprised the group without CZS and had higher HRV than those in the CZS group.

Our results should be interpreted in light of its limitations, such as the small sample size, and its strengths, which are the novelty of the information and the importance of proposing a sentinel event for the prediction of worsening neurological evolution and the possibility of cardiovascular comorbidities in patients with antenatal exposure to ZIKV. Due to the absence of normal HRV values for patients of the age in our study, we chose to describe the values in this group of infants with antenatal exposure to ZIKV and compare the values among subgroups of the sample.

\section{Conclusions}

This study is unprecedented in a population of infants of this age and with antenatal exposure to ZIKV. Given the seasonality of arbovirus infection, we cannot predict when it will be possible to repeat the study in a larger sample, but our findings suggest that 24-hour Holter monitoring can be used early in newborns and young infants with a history of intrauterine exposure to ZIKV and born without full spectrum CZS, to indicate the early initiation of rehabilitation therapies such as occupational therapy, speech therapy, physiotherapy and psychomotor therapy. In addition, it calls attention to the development of possible comorbidities, such as cardiovascular diseases, that may present in the medium/long term and require early prevention measures.

\section{Abbreviations}

ZIKV: Zika virus

CNS: Central nervous system

CZS: Congenital Zika syndrome

ANS: Autonomic nervous system

SIDS: Sudden infant death syndrome

HR: Heart rate

HRV: Heart rate variability

SDNN: Standard deviation of R-R intervals throughout the Holter "clean trace" time

Pnn50: The mean number of times per hour when the R-R intervals exceed 50 milliseconds

rMMSD: The mean square root of successive differences between normal heart beats 
PCR: Polymerase chain reaction

PT: Preterm

SGA: Small for gestational age

HP: Head perimeter

SD: Standard deviation

\section{Declarations}

Ethics approval and consent to participate: The study was approved by the Research Ethics Committee of the National Institute of Infectology (INI-FIOCRUZ), and all the parents and guardians agreed to sign the informed consent form.

Availability of data and materials: The datasets used and/or analysed during the current study are available from the corresponding author on reasonable request.

Competing interest: Authors declared no competing interests.

Funding: SRLP is supported by Capes (Grant number $88881130793 / 2016-1)$, CNPq (grants number440846/2016-1 and 310765/2016-1) and has a Research Productivity Scholarship-UNESA).

Authors' contributions:

DHGO: conception of the study, design of the study, acquisition of data, analysis of data, draft the manuscript.

SRLP: conception of the study, design of the study, substantially revised the work.

SMP,MVSP,EBA,IOA: acquisition of data.

TMR,LMLS,BM,LNS,RVG,RAO: acquisition of data, analysis of data.

MEM: conception of the study.

RVCO: conception and design of the study,analysis of data, substantially revised the work.

\section{References}

1. Meneses JDA, Ishigami AC, de Mello LM, de Albuquerque LL, de Brito CAA, Cordeiro MT, Pena LJ. Lessons Learned at the Epicenter of Brazil's Congenital Zika Epidemic:

Evidence From 87 Confirmed Cases.Clin Infect Dis. 2017 May 15;64(10):1302-1308. doi: 
10.1093/cid/cix166.

2. Moore CA, Staples JE, Dobyns WB, Pessoa A, Ventura CV, Fonseca EB et al. Characterizing the Pattern of Anomalies in Congenital Zika Syndrome for Pediatric Clinicians. JAMA Pediatr. 2017. March 1;171(3):288-295.

doi:10.1001/jamapediatrics.2016.3982

3. Orofino DHG, Passos SRL, de Oliveira RVC, Farias CVB, Leite MdFMP, Pone SM, et al. (2018) Cardiac findings in infants with in utero exposure to Zika virus- a cross sectional study. PLoS Negl Trop Dis. 2018Mar 26;12(3): e0006362. doi:

10.1371/journal.pntd.0006362. eCollection 2018 Mar

4. Cavalcanti DD, Alves LV, Furtado GJ, Santos CC, Feitosa FG, Ribeiro MC, et al. Echocardiographic findings in infants with presumed congenital Zika syndrome: Retrospective case series study. PLOS ONE 12(4): e0175065. https://doi.org/10.1371/journal.pone.0175065 PMID: 28426680

5. Galland BC, Taylor BJ, Bolton DP, Sayers RM. Heart rate variability and cardiac reflexes in small for gestational age infants.J Appl Physiol (1985). 2006 Mar;100(3):933-9. Epub 2005 Nov 23.

6. Rakow A, Katz-Salamon M, Ericson M, Edner A, Vanpée M. Decreased heart rate variability in children born with low birth weight. Pediatr Res. 2013 Sep;74(3):339-43. doi:

10.1038/pr.2013.97. Epub 2013 Jun 14.

7. Franciosi S, Perry FKG, Roston TM, Armstrong KR, Claydon VE, Sanatani S. The role of the autonomic nervous system in arrhythmias and sudden cardiac death. Auton Neurosci. 2017 Jul;205:1-11. doi: 10.1016/j.autneu.2017.03.005. Epub 2017 Mar 31.

8. Shaffer F, McCraty R, Zerr CL. A healthy heart is not a metronome: an integrative review of the heart's anatomy and heart rate variability. Front Psychol. 2014; 5: 1040. Published online 2014 Sep 30. doi: $10.3389 /$ fpsyg. 2014.01040

9. Massin M,von Bernuth G. Normal Ranges of heart rate variability during infancy and childhood.Pediatr Cardiol. 1997 Jul-Aug; 18(4): 297-302.

10. Vanderlei LCM, Pastre CM, Hoshi RA, Carvalho TD de, Godoy MF de. Basic notions of heart rate variability and its clinical applicability. Rev Bras Cir Cardiovasc. 2009 AprJun;24(2):205-17.

11. Schechtman VL, Harper RM, Kluge KA, Wilson AJ, Hoffman HJ, Southall DP. Cardiac and respiratory patterns in normal infants victims of sudden infant death syndrome. Sleep 11:413-424.Sleep. 1988 Oct;11(5):413-24

12. Fox N,Porges S. The relation between neonatal period patterns and development outcome. 
Child Dev 1985; 56:28-37.

13. Doussard- Roosevelt J, Porges S, Scanlon J, Alemi B, Scanlon K.Vagal regulation of heart rate in the prediction of developmental outcome for very low birth weight preterm infants.

Chil Dev 1997; 68:173-86.

14. Baysal-Kirac L, Serbest NG, Şahin E, Dede HÖ, Gürses C, Gökyiğit A, Bebek N, Bilge AK, Baykan B. Analysis of heart rate variability and risk factors for SUDEP in patients with drug-resistant epilepsy. Epilepsy Behav. 2017 Jun;71(Pt A):60-64. doi: 10.1016/j.yebeh.2017.04.018. Epub 2017 May 23.

15. Rabe IB, Staples JE, Villanueva J, Hummel KB, Johnson JA, Rose L; MTS, Hills S,

Wasley A, Fischer M, Powers AM. Interim Guidance for Interpretation of Zika Virus Antibody Test Results. MMWR Morb Mortal Wkly Rep. 2016 Jun 3;65(21):543-6. doi:

10.15585/mmwr.mm6521e1.

16. The Apgar Score. Pediatrics. 2015; 136:819-822. American Academy of Pediatrics Committee on Fetus and Newborn; American College of Obstetricians and Gynecologists Committee on Obstetric Practice.

17. http://www.who.int/es/news-room/fact-sheets/detail/preterm-birth (último acesso em 12/11/2018)

18. Villar J, Cheikh Ismail L, Victora CG, et al. International standards for newborn weight, length, and head circumference by gestational age and sex: the Newborn Cross-Sectional Study of the Intergrowth21st Project. Lancet. 2014; 384:857-68.

19. Malik M. Heart rate variability: standards of measurement,physiological interpretation and clinical use.Task Force of the Eueropean Society of Cardiology and the North American Society of Pacing and Eletrophysiology. Circulation1996; 93:927-34.

20. Ronald L. Wasserstein \& Nicole A. Lazar (2016) The ASA's Statement on $p$-Values: Context, Process, and Purpose, The American Statistician, 70:2, 129-133,DOI: 10.1080/00031305.2016.1154108

21.Bjelakovic B, Ilic S, Dimitrijevic L, Milovanovic B, Kostic G, Bjelakovic L, Lukic S. Heart rate variability in infants with central coordination disturbance. Early Hum Dev. 2010 Feb;86(2):77-81. doi:

10.1016/j.earlhumdev.2010.01.022. Epub 2010 Feb 9.

22.Schechtman VL, Raetz SL, Harper RK, Garfinkel A, Wilson AJ, Southall DP, Harper RM. Dynamic analysis of cardiac R-R intervals in normal infants and in infants who subsequently succumbed to the sudden infant death syndrome.Pediatr Res. 1992 Jun;31(6):606-12. 